\title{
Mixed Sampling Product Control for Costly or Destructive Items
}

\author{
S. Deva Arul ${ }^{*}$ and K. Rebecca Jebaseeli Edna ${ }^{2}$ \\ 1 Department of Statistics, Government Arts College, Coimbatore, India. ${ }^{2}$ Department of \\ Mathematics, Karunya University, Coimbatore, India. \\ *Corresponding author: devaarul_s@rediffmail.com.
}

Received: July 31, 2011 / Accepted: August 19, 2011

\begin{abstract}
This paper presents the derivation of operating characteristics function and associated measures of performance of mixed sampling Plans for costly or destructive items. The first stage is concerned with single sample variable inspection and in the second stage modified Chain Sampling inspection is recommended. If the first stage inspection of mixed plans does not lead to acceptance of the batch or lot then the second stage becomes more important. In the second stage of mixed plans, it is advisable to have small sample size for costly or destructive items. Therefore, in the second stage, the modified chain sampling plan which always utilizes the past lot inspection results in order to achieve very small sample size is taken into consideration. The economic or cost-effective of the sampling plans is measured through Average Sample Number which is smaller compared to any other mixed sampling plans. Designing procedure is given and tables are constructed to facilitate easy application in the production industries.
\end{abstract}

Keywords: Mixed Sampling, OC function (Operating Characteristics), AQL(Acceptable Quality Level), I stage Sampling, II stage Sampling, Variable sampling, Modified Chain Sampling. 


\section{Introduction}

Mixed Sampling Plans was introduced by Dodge (1957). Later Schilling (1967) has given a methodology to determine the Operating characteristics function and its associated measures. Mixed Sampling Plan consists of two stages. The first stage deals with variable criteria and the second stage is considered with attribute criteria. In a Mixed Sampling Plans, the second stage of attribute inspection becomes more important to discriminate the lot, if the first stage variable inspection fails to accept the lot. In the second stage of mixed plans, it is advisable to have small sample size for costly or destructive items. So in the second stage, acceptance number of zero plans is more emphasized for practical reason. However the $O C$ curve of acceptance number with zero plans does not discriminate between good and bad lots. Hence Chain Sampling is recommended in the second stage of mixed plans. But Chain sampling plans in the second stage does not guarantee small sample sizes. Therefore modified chain sampling plans are recommended in the second stage. Several measures of Modified Mixed Sampling Plans are derived and algorithm is given to sentence a lot or batch. This article deals with the operating procedure of mixed acceptance sampling plans with modified chain sampling as attribute plan. The operating characteristics function and other associated measures of the plan are derived and given. The resulting plans yield small sample size in both the stages. These mixed plans are costeffective with respect to sampling inspection.

DevaArul (2004) has developed mixed sampling plans by inculcating the blend of process and product control measures to suit industrial needs. DevaArul (2009) has contributed towards mixed sampling system with tightened inspection in the second stage. Suresh and DevaArul (2002) have developed mixed sampling plans by combining process and product quality characteristics to reduce the sampling cost. Dodge E.G (1955) has developed 'Chain Sampling Inspection Plan'. Govindaraju and Lai (1998) have developed modified chain sampling plans for costly or destructive items. In this paper the blend of concepts from Govindaraju and Lai (1998) and Schilling (1967) have been studied and modified to suite mixed sampling plans. 


\section{Materials and Methods:}

\section{Formulation of Mixed Sampling Plan with Modified Chain Sampling Plan as Attribute Plan}

The development of mixed plans and the subsequent discussion are limited only to the upper specification limit. By symmetry a parallel discussion can be made use for lower specification limits. It is suggested that the mixed plans with modified chain sampling as attribute plan in the case of single sided specification (U), standard deviation known can be formulated by the four parameters $n_{1}, n_{2}, k$ and $i$, where, $n_{1}=$ First Sample Size, $n_{2}=$ second sample size.

$$
\begin{aligned}
& \mathrm{k}=\text { Variable Factor such that a lot is accepted if } \bar{x} \leq U-k \sigma \\
& \mathrm{U}=\text { Upper Specification Limit. } \\
& \mathrm{n}_{2}=\text { Second Sample Size. } \\
& \mathrm{N}=\text { Lot size. } \\
& \bar{x}=\text { Sample Mean } \\
& \sigma=\text { Population Standard Deviation. } \mathrm{i}=\text { index of chaining the lots }
\end{aligned}
$$

\section{Algorithm for Sentencing a Lot}

Let the two stages be independent.

Step (i) Determine the four parameters usually with reference to ASN and OC curves

Step (ii) Take a random sample of size $n_{1}$ from the lot.

Step (iii) If the sample average $\bar{x} \leq A=U-k \sigma$, accept the lot.

Step (iv) If the sample average $\bar{x}>A$ take a second sample of size $n_{2}$.

Step ( $v$ ) Inspect and find the number of defectives (d) in the second sample.

i) Accept the lot if

1. the current sample as well as the preceding 'i' samples contain no nonconfirming units.

2. the current sample contains no nonconfirming units, while any one of the ' $i$ ' preceding samples contain only one nonconfirming unit and the rest of (i-1) samples are free from non-confirming units

ii) Otherwise reject the lot. 


\section{Measures of Independent Plan}

In this section various kinds of Measures of Sampling Plans relevant to the Mixed Sampling Plans are derived and given.

I. Probability of acceptance

$$
\begin{aligned}
& P_{a}(p)=P_{n_{1}}(\bar{x} \leq A)+P_{n_{1}}(\bar{x}>A) P_{0}\left(P_{0}^{i}+i P_{0}^{i-1} P_{1}\right) \ldots \\
& P_{0}=e^{-n_{2} p} \text { and } P_{1}=n_{2} p e^{-n_{2} p} \\
& P_{a}(p)=P_{n_{1}}(\bar{x} \leq A)+P_{n_{1}}(\bar{x}>A) e^{-n_{2} p(i+1)}\left(1+i n_{2} p\right)
\end{aligned}
$$

II. Average sample number:

$\mathrm{ASN}=n_{1}+n_{2} P(\bar{x}>A)$

III. Average Total Inspection

$\mathrm{ATI}=\mathrm{ASN}+\left(\mathrm{N}-n_{1}-n_{2}\right)\left(1-P_{a}(\mathrm{p})\right)$

IV. Average outgoing quality:

$A O Q=p \cdot P_{a}(p)$

\section{Designing Modified Mixed Sampling Plans Indexed Through AQL}

Designing the mixed sampling plan with variable Single sampling plans in the first stage and modified chain sampling plan as attribute plan in the second stage, when $\left(p_{1}, \beta_{1}=L\left(p_{1}\right)\right)$, $i$ and the first sample size $n_{1}$ are known. Assume that problem of acceptance assigned to the attribute plan associated with the second stage sample as the mixed plans are independent

1. Split the probability of acceptance that will be assigned to the first stage. Let it be $\beta_{1}^{\prime}$.

2. Decide the sample size $n_{1}$, which is to be used.

3. Calculate the acceptance limit as

$$
A=U-\left[Z\left(p_{1}\right)+\frac{Z\left(\beta_{1}^{\prime}\right)}{\sqrt{n_{1}}}\right] \sigma
$$

4. Now determine $\beta_{1}$ " the probability of acceptance assigned to the attribute plan associated with the second stage sample as, 


$$
\beta_{1}^{\prime \prime}=\frac{\beta_{1}-\beta_{1}^{\prime}}{1-\beta_{1}^{\prime}}
$$

5. Determine the appropriate second stage sample of size $n_{2}$ and index $i$ from the relation.

$$
e^{-n_{2} p(i+1)}\left(1+i n_{2} p\right)=\beta_{1}
$$

The above equation cannot be solved easily. Hence the solutions are obtained by using an iterative procedure. A computer program is written to solve the equation and tables are constructed to facilitate easy application at shaft floors.

\section{Designing Modified Mixed Sampling Plans Indexed Through AQL and LQL}

.Assume that the plan is to be independent.

2.Split the probability of acceptance that will be assigned to the first stage. Call these as $\beta_{1}^{\prime}$ and $\beta_{2}^{\prime}$ respectively. Note that $\beta_{1} \geq \beta_{1}^{\prime}$ and $\beta_{2} \geq \beta_{2}^{\prime}$

3.Use the standard variable procedure, determine the first sample size $n_{1}$ as

$$
n_{1}=\left[\frac{Z\left(\beta_{2}{ }^{\prime}\right)-Z\left(\beta_{1}{ }^{\prime}\right)}{Z\left(p_{1}\right)-Z\left(p_{2}\right)}\right]^{2}
$$

4. Then the acceptance limit is

$$
A=U-\left[Z\left(p_{1}\right)+\frac{Z\left(\beta_{1}^{\prime}\right)}{\sqrt{n_{1}}}\right] \sigma
$$

5. Now determine the probability of them acceptance associated with $p_{1}$ and $p_{2}$ respectively. Let them be $\beta_{1} "$ and $\beta_{2}{ }^{\prime \prime}$

$$
\begin{aligned}
& \beta_{1}^{\prime \prime}=\frac{\beta_{1}-\beta_{1}^{\prime}}{1-\beta_{1}^{\prime}} \\
& \beta_{2}^{\prime \prime}=\frac{\beta_{2}-\beta_{2}^{\prime}}{1-\beta_{2}^{\prime}}
\end{aligned}
$$

6. Determine the appropriate second sample size $n_{2}$ and the acceptance number $i$, such that $e^{-n_{2} p(i+1)}\left(1+i n_{2} p\right)=\beta_{1} \quad$ for fraction defective $p=p_{1}$ $e^{-n_{2} p(i+1)}\left(1+i_{2} p\right)=\beta_{2}{ }^{\prime} \quad$ for fraction defective $\mathrm{p}=\mathrm{p}_{2}$ 


\section{Construction of Tables}

Solving the above non-linear equations manually is not simple. Based on the Designing procedure a Computer Program is written to determine the sampling plan parameters.

Table 1: Values of the first stage variable criteria ' $k$ ' and the second stage sample size $\mathrm{n}_{2}$ for the known AQL (Using modified chain sampling plan as attribute plan in the second stage). $\beta_{1}=0.95$, $\beta_{1}^{\prime}=0.65$.

\begin{tabular}{|l|l|l|l|l|l|l|l|l|l|l|l|l|l|l|l|}
\hline \multirow{2}{*}{$p$} & \multicolumn{10}{|c|}{ Values of $\mathrm{n}_{2}$} \\
\cline { 2 - 7 } & $\mathrm{i}=1$ & 2 & 3 & 4 & 5 & 6 & 7 & 8 & 9 & 10 & $\mathrm{n}_{1}=5$ & $\mathrm{n}_{1}=10$ & $\mathrm{n}_{1}=15$ & $\mathrm{n}_{1}=20$ \\
\hline 0.001 & 100 & 100 & 100 & 92 & 79 & 70 & 64 & 58 & 53 & 49 & 2.933 & 2.98 & 3.002 & 3.015 \\
\hline 0.002 & 66 & 58 & 52 & 45 & 40 & 35 & 32 & 29 & 27 & 25 & 2.713 & 2.76 & 2.782 & 2.795 \\
\hline 0.003 & 44 & 39 & 34 & 30 & 27 & 24 & 22 & 20 & 18 & 17 & 2.583 & 2.63 & 2.652 & 2.665 \\
\hline 0.004 & 33 & 29 & 26 & 23 & 20 & 18 & 16 & 15 & 15 & 14 & 2.483 & 2.53 & 2.552 & 2.565 \\
\hline 0.005 & 27 & 24 & 21 & 18 & 16 & 14 & 13 & 12 & 11 & 10 & 2.403 & 2.45 & 2.472 & 2.485 \\
\hline 0.006 & 22 & 20 & 17 & 15 & 14 & 12 & 11 & 10 & 9 & 8 & 2.343 & 2.39 & 2.412 & 2.425 \\
\hline 0.007 & 19 & 17 & 15 & 13 & 11 & 10 & 9 & 9 & 8 & 7 & 2.283 & 2.33 & 2.352 & 2.365 \\
\hline 0.008 & 17 & 15 & 13 & 12 & 10 & 9 & 8 & 8 & 7 & 7 & 2.243 & 2.29 & 2.312 & 2.325 \\
\hline 0.009 & 15 & 13 & 11 & 10 & 9 & 8 & 7 & 7 & 6 & 6 & 2.193 & 2.24 & 2.262 & 2.275 \\
\hline 0.01 & 14 & 12 & 11 & 9 & 8 & 7 & 7 & 6 & 5 & 5 & 2.153 & 2.2 & 2.222 & 2.235 \\
\hline 0.015 & 9 & 8 & 7 & 6 & 6 & 5 & 5 & 4 & 4 & 4 & 2.003 & 2.05 & 2.072 & 2.085 \\
\hline
\end{tabular}

\section{Example 1:}

Suppose a production process turns out $1 \%$ defective. Obtain the corresponding mixed sampling plan with modified chain sampling as attribute plan with Probability of acceptance as $95 \%$ and the past lot index $i=5$ with first stage sample size is 5 .

\section{Solution:}

It is given that the probability of acceptance is $95 \%$. Let us bifurcate the $1^{\text {st }}$ stage probability of acceptance. $\beta_{1}=0.65$; Then the second stage probability of acceptance will be $\beta_{1}^{\prime \prime}=0.86$.

Suppose if the first stage sample size $n_{1}=5$ then from table $1, n_{2}=8$. Hence the parameters of mixed sampling plan with modified chain sampling are $n_{1}=5, n_{2}=8, i=5$ and $k=2.933$. 


\section{Table 2}

The mixed sampling plans indexed through AQL and LQL when $\left(p_{1}, \beta_{1}\right)$ and $\left(p_{2}, \beta_{2}\right)$ are known.

Assuming $\beta_{1}=0.95, \beta_{2}=0.05, \beta_{1}^{\prime}=0.65, \beta_{2}{ }^{\prime}=0.02$.

\begin{tabular}{|c|c|c|c|c|c|c|c|c|c|c|c|c|c|}
\hline \multirow[t]{2}{*}{$p_{1}$} & \multirow[t]{2}{*}{$p_{2}$} & \multirow[t]{2}{*}{$\mathrm{n}_{1}$} & \multirow[t]{2}{*}{$\mathrm{k}$} & \multicolumn{10}{|c|}{ Values of $n_{2}$} \\
\hline & & & & $i=1$ & $i=2$ & $i=3$ & $i=4$ & $i=5$ & $i=6$ & $i=7$ & $i=8$ & $i=9$ & $i=10$ \\
\hline .001 & .01 & 10 & 2.9798 & 153 & 135 & 127 & 90 & 80 & 72 & 65 & 58 & 53 & 48 \\
\hline .002 & .02 & 9 & 2.7533 & 90 & 72 & 58 & 48 & 42 & 36 & 32 & 29 & 27 & 24 \\
\hline .003 & .03 & 8 & 2.6156 & 65 & 50 & 38 & 32 & 28 & 24 & 22 & 20 & 18 & 16 \\
\hline .004 & .04 & 7 & 2.5064 & 50 & 38 & 29 & 24 & 21 & 18 & 16 & 15 & 14 & 13 \\
\hline .005 & .05 & 6 & 2.4231 & 40 & 28 & 23 & 19 & 17 & 14 & 13 & 12 & 11 & 10 \\
\hline .006 & .06 & 6 & 2.3528 & 34 & 24 & 19 & 16 & 14 & 12 & 11 & 10 & 9 & 8 \\
\hline .007 & .07 & 6 & 2.3032 & 29 & 20 & 17 & 13 & 12 & 10 & 9 & 8 & 8 & 7 \\
\hline .008 & .08 & 5 & 2.238 & 25 & 19 & 14 & 12 & 10 & 9 & 8 & 8 & 7 & 6 \\
\hline .009 & .09 & 5 & 2.198 & 23 & 16 & 13 & 11 & 9 & 8 & 7 & 7 & 6 & 5 \\
\hline .010 & .10 & 5 & 2.148 & 20 & 15 & 12 & 10 & 8 & 7 & 7 & 6 & 5 & 5 \\
\hline
\end{tabular}

\section{Example 2:}

Let $p_{1}=0.005, p_{2}=0.05$ and the corresponding probability of acceptance be $\beta_{1}=0.95, \beta_{2}=$ 0.05 obtain the mixed plan with modified chain sampling plan as attribute plan when $\mathrm{i}=3$

\section{Solution:}

By assuming $\beta_{1}{ }^{\prime}=0.65, \beta_{2}{ }^{\prime}=0.02$, from Table (2), the parameters are $n_{1}=6, k=2.4231$ and $\mathrm{n}_{2}=23$ for $\mathrm{i}=3$. 


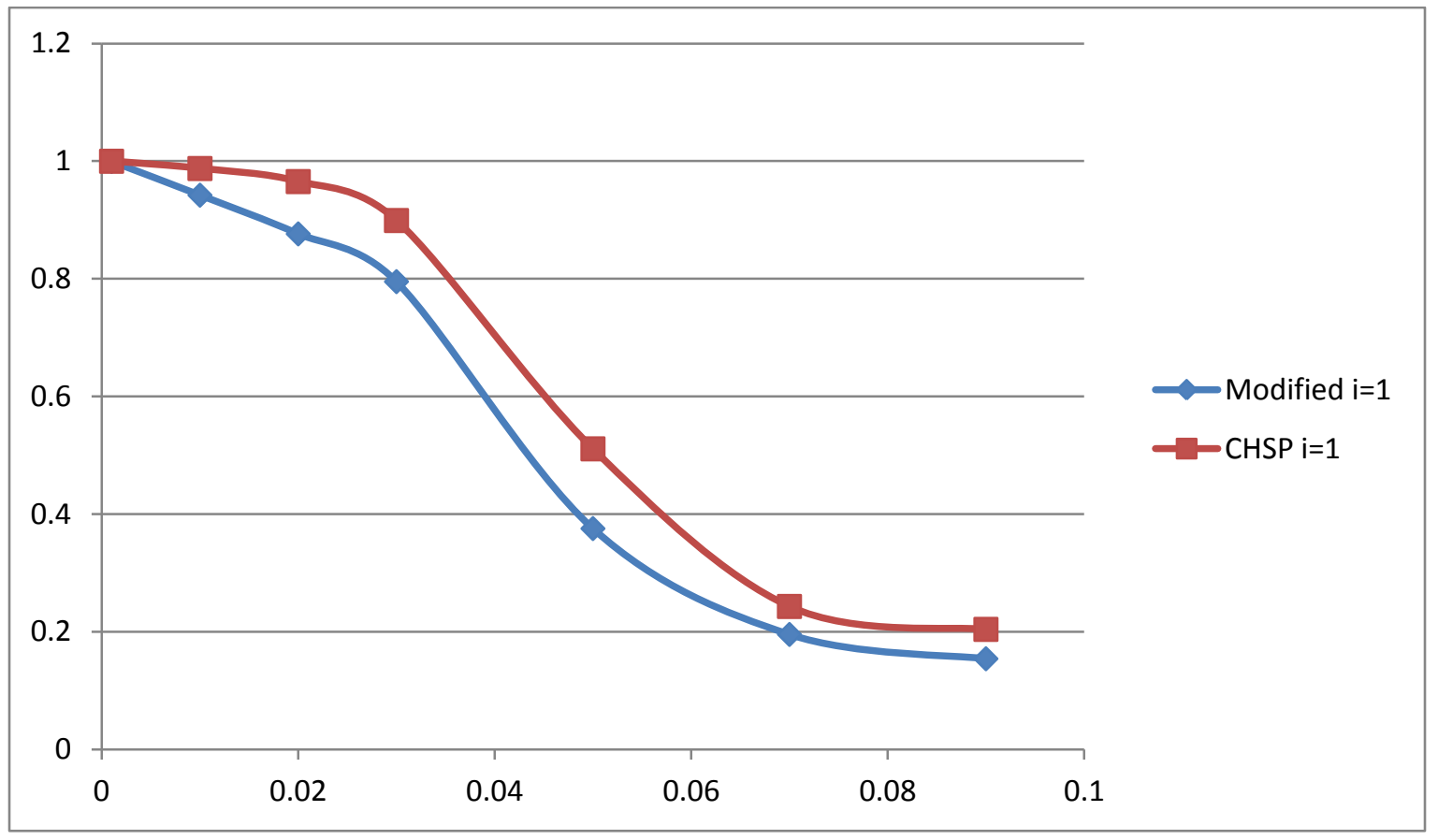

Figure (i). Comparison of $O C$ curves with index $i=1$.

Table 3: Comparison of ASN values for $\mathrm{i}=1$.

\begin{tabular}{|l|l|l|l|l|}
\hline $\begin{array}{l}\text { Mixed Modified chain sampling plan as } \\
\text { attribute plan in the second stage } \mathrm{n}_{1}=5, \\
\mathrm{n}_{2}=15, \mathrm{i}=1\end{array}$ & $\begin{array}{l}\text { Mixed Chain sampling plan as attribute } \\
\text { plan in the second stage } \mathrm{n}_{1}=5 \mathrm{n}_{2}=41, \\
\mathrm{i}=1\end{array}$ \\
\hline $\mathrm{p}$ & $\mathrm{P}_{\mathrm{a}}$ & ASN & $\mathrm{P}_{\mathrm{a}}$ & ASN \\
\hline 0.001 & 0.99967 & 5 & 0.99995 & 6 \\
\hline 0.002 & 0.99815 & 6 & 0.99944 & 8 \\
\hline 0.003 & 0.99525 & 7 & 0.99794 & 9 \\
\hline 0.004 & 0.99173 & 7 & 0.99489 & 11 \\
\hline 0.005 & 0.9850 & 8 & 0.9901 & 13 \\
\hline
\end{tabular}




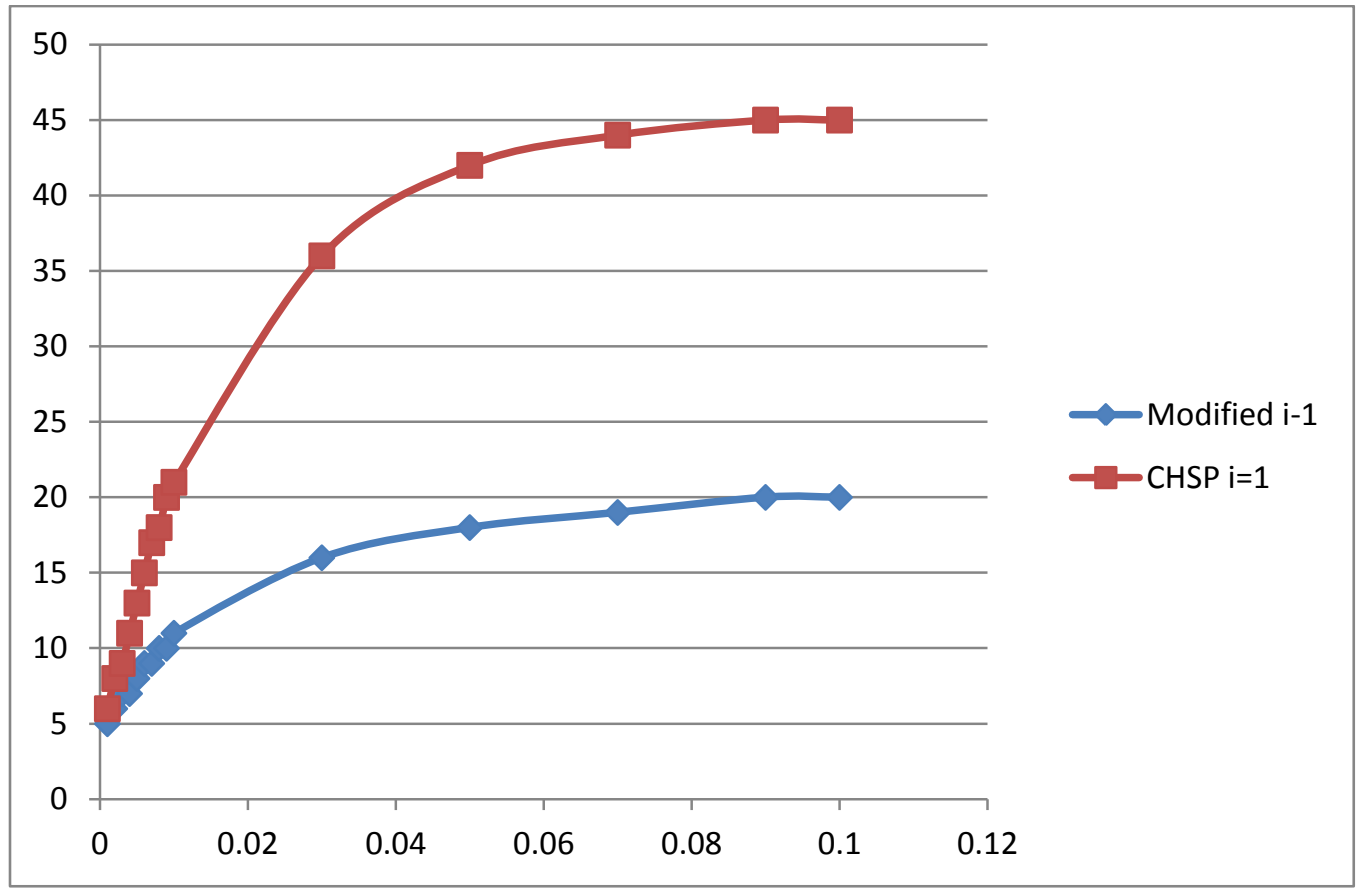

Figure (ii). Comparison of ASN Curves using table 3.

\section{Results and Discussion}

\section{Comparison between the Sampling Plans}

From fig (ii), one can find that for the same set of Probability of acceptance, the sample sizes are lesser for newly proposed MIXED plans when compared to the mixed sampling plans with Chain sampling as attribute plan. It is interesting to note that when ' $i$ ' increases, the Operating characteristics curve tends to be ideal OC curve.

\section{Conclusion}

This paper presents derivation of operating characteristics and associated performance measures of mixed sampling plans for costly or destructive items. The operating procedure and designing of the mixed sampling plans are given. The first stage is considered with single sampling inspection due to variable criteria and the second stage is concerned with attribute inspection. The resulting sampling plans yields small sample sizes. Hence the economic average sample number is attained. 
Tables are constructed to facilitate easy selection of mixed plans. The comparative values of ASN clearly indicate the reduction in the sample size in the proposed mixed plans.

\section{Acknowledgements}

The authors are thankful to Dr. Paul Dhinakaran, Chancellor, Karunya University, Coimbatore, for his kind hearted support in Research Endeavour.

\section{References}

[1] DevaArul S (2004) Certain Studies relating to Mixed Sampling plans and Reliability based Sampling Plans. Ph.D. Thesis, Bharathiar University, India.

[2] DevaArul S and K Rebecca Jebaseeli Edna (2010) Dependent Multi-dimensional Mixed Sampling Plans International Journal of Applied Mathematics and Computation 8(S10):28-38.

[3] DevaArul S (2009) Mixed sampling system with tightened inspection in the second stage. International Journal of Artificial Intelligence 2(S09):57-65.

[4] DevaArul S and V Jemmy Joyce (2010) Mixed Sampling Plans for second quality lot. Economic Quality Control 25(1):31-42.

[5] Dodge HF (1955) Chain Sampling Inspection Plan.Industrial Quality Control 11 (4):10-13.

[6] Govindaraju K and C.D Lai (1998) A modified Chsp-1 Chain Sampling Plan,MChsp-1, with very Small Sample Sizes. American Journal of Mathematical and Management Sciences 18(3-4):343-358.

[7] Schilling E.G(1967) A general method for determining the OC of Mixed variableattributes Sampling plans, Single sided specifications, SD known. PhD Thesis, Rutgers, New Jersey.

[8] Suresh K.K and DevaArul S(2002) Designing and Selection of Mixed Sampling Plans with Chain Sampling as attribute plan. Quality Engineering Journal, 15(1):155-160.

[9] Suresh K.K and DevaArul S(2002) Combining Process and Product Control for Reducing Sampling Cost. Economic Quality Control, Journal and Newsletter for Quality and Reliability 17(2):187-194. 